Published in final edited form as:

Horm Behav. 2015 November ; 76: 3-10. doi:10.1016/j.yhbeh.2015.04.013.

\title{
Surprising Origins of Sex Differences in the Brain
}

\author{
Margaret M. McCarthy, Lindsay A. Pickett, Jonathan W. VanRyzin, and Katherine E. Kight \\ Department of Pharmacology, Program in Neuroscience and Program in Molecular Medicine, \\ University of Maryland School of Medicine, Baltimore MD 21201
}

\section{Abstract}

Discerning the biologic origins of neuroanatomical sex differences has been of interest since they were first reported in the late 60's and early 70's. The centrality of gonadal hormone exposure during a developmental critical window cannot be denied but hormones are indirect agents of change, acting to induce gene transcription or modulate membrane bound signaling cascades. Sex differences in the brain include regional volume differences due to differential cell death, neuronal and glial genesis, dendritic branching and synaptic patterning. Early emphasis on mechanism therefore focused on neurotransmitters and neural growth factors, but by and large these endpoints failed to explain the origins of neural sex differences. More recently evidence has accumulated in favor of inflammatory mediators and immune cells as principle regulators of brain sexual differentiation and reveal that the establishment of dimorphic circuits is not cell autonomous but instead requires extensive cell-to-cell communication including cells of non-neuronal origin. Despite the multiplicity of cells involved the nature of the sex differences in the neuroanatomical endpoints suggests canalization, a process that explains the robustness of individuals in the face of intrinsic and extrinsic variability. We propose that some neuroanatomical endpoints are canalized to enhance sex differences in the brain by reducing variability within one sex while also preventing the sexes from diverging too greatly. We further propose mechanisms by which such canalization could occur and discuss what relevance this may have to sex differences in behavior.

The origins of behavioral differences between men and women, boys and girls, males and females, has been a topic of fascination since the dawn of consciousness. In contrast, the idea that the brain is the principle means by which males and females behave differently is strikingly modern, having only been accepted as a possibility following a Battle of the Titans in the 1950's when Frank Beach famously argued that the critical variable that determined how males behaved versus females was the type of genitalia one possessed, either intromitting or receiving (Beach 1974, Phoenix et al 1959). This viewpoint was eventually overturned beginning with an iconic paper published in 1959. Using guinea pigs as a model William C. Young and colleagues convincingly demonstrated that prenatal hormones were capable of sex reversing the behavior of females when adults (Phoenix et al

Corresponding Author: Margaret M McCarthy, PhD, University of Maryland School of Medicine, 655 W. Baltimore St, Baltimore MD 21201, mmccarth@umaryland.edu.

Publisher's Disclaimer: This is a PDF file of an unedited manuscript that has been accepted for publication. As a service to our customers we are providing this early version of the manuscript. The manuscript will undergo copyediting, typesetting, and review of the resulting proof before it is published in its final citable form. Please note that during the production process errors may be discovered which could affect the content, and all legal disclaimers that apply to the journal pertain. 
1959). While this and other studies succeeded in ending the debate about what organ in the body was controlling sex differences in behavior, it also generated a degree of tunnel vision as the next 2-3 decades were dominated by studies of reproductive behavior and physiology leading to the widespread belief that sex differences in the brain are narrow in both their scope and significance, being limited to control of the anterior pituitary gland, courtship, copulation and parenting.

Hormonal modulation of neural plasticity opened the gateway for sex differences outside the context of reproduction. This can largely be traced to the seminal finding of the McEwen lab that dendritic spine density on hippocampal pyramidal neurons varied by almost $30 \%$ across the few days of the estrus cycle in female rats (Woolley \& McEwen 1992). This was viewed as an astonishing level of plasticity at that time and initial reports were met with skepticism. But again, an irrefutable march of data led to the general acceptance that indeed hormones are powerful regulators of neuronal function outside of the diencephalon and outside the context of reproduction. Importantly, however, modulation of adult neural function or behavior by hormones is not the same as sex differences in neural function or behavior. Investigation into whether adult functions known to be impacted by steroids in a modulatory manner are sexually differentiated are actually relatively few. For instance, estradiol alters synaptic physiology and cognitive function in adult females, but are these endpoints also subject to sexual differentiation? Answering this question is actually surprisingly difficult precisely because of the hormonal modulation in adulthood. What does one compare? An estrous female to an intact male or maybe both sexes should be gonadectomized and hormone replaced to standardize their endocrinology? But whose endocrine profile do you choose? It quickly becomes a Gordian Knot of possibilities and scares off even the bravest of the curious. Further complicating the picture is the impact of a lifetime of experience and environment which can vary in profound and significant ways between males and females. These difficulties can be avoided, however, by studying the origins of sex differences in development, which while not immune to environment and experience, are at least somewhat buffered from them simply by not having had as much time to have accumulated an effect.

\section{Historical Perspective on Sex Differences in the Brain}

The first robust sex difference discovered in the healthy mammalian brain was aptly named the sexually dimorphic nucleus of the preoptic area (SDN-POA) (Gorski et al 1978, Gorski et al 1980) and is one of, if not the most, extensively studied sex difference in the brain. It is located under the anterior commissure, above the optic chiasm, lateral to the third ventricle, and anterior to the hypothalamus (Figure 1). Neurogenesis in the SDN begins at embryonic day 14 (E14) and ends on E18. The sensitive period during which the size of the SDN is influenced by gonadal steroids is from E18 to postnatal day 4 (PN4) (Jacobson \& Gorski 1981, Orikasa et al 2010, Rhees et al 1990a, Rhees et al 1990b). Although first described as a densely-packed bundle of neurons revealed with a simple Nissl stain (Gorski et al 1978, Gorski et al 1980), the SDN has since been more clearly (and reliably) defined by a subset of cells that are immunopositive for the expression of Calbindin-D28k (a calcium binding protein expressed in GABAergic neurons and implicated in neuroprotection) and is thus now referred to as the CALB-SDN (Kato et al 2012, Sickel \& McCarthy 2000). Sex differences 
in the SDN and the CALB-SDN, are of the same magnitude and both arise after PN4. During development, the SDN of males and females have similar numbers of neurons but due to higher levels of apoptotic cells undergoing DNA-fragmentation in females between PN6 and PN9, this region becomes markedly smaller in females (Davis et al 1996). Other studies support the role of apoptosis in sexual differentiation of the SDN by demonstrating that the male SDN has higher expression of anti-apoptotic Bcl-2 (at the protein but not mRNA level) while the female SDN contains higher expression of pro-apoptotic Bax from PN5-PN7. Apoptosis via caspase-3 activation occurs at higher rates in the female SDN and this corresponds with the decrease in size of the female SDN. Sex differences in proapoptotic Bax and Bcl-2 are abolished in response to treatment of females with estradiol but the mechanism by which estradiol modulates Bcl-2 and Bax in the SDN is not yet clear (Tsukahara et al 2008, Tsukahara et al 2006). Interestingly, when sex differences in the CALB-SDN were investigated in a Bax knockout mouse, there were no significant increases in the size of the CALB-SDN as compared to their wild type counterparts, suggesting that Bax expression is not the primary mechanism by which cells are dying off in females (Gilmore et al 2012). A role for estradiol up regulation of calbindin and calretinin expression in males has been considered but not clearly demonstrated. Elevated levels of these calcium binding proteins may protect cells from toxicity that can result from excessive neuronal excitation in males compared to females. Although testosterone treatment can up regulate calbindin and calretinin expression in the hypothalamus (Brager 2000, Watson et al 1998), this has not been demonstrated specifically in the SDN. Thus, despite much speculation over mechanisms by which estradiol regulates volume of the SDN, no consensus has been reached and the origins of this iconic sex difference remain a mystery.

Subsequent to the discovery of the SDN in the rodent, analogous structures were reported in the ferret (Baum et al 1996, Park et al 1996), sheep (Roselli et al 2004) and primates, including humans(Hofman \& Swaab 1989, LeVay 1991, Swaab \& Hofman 1995). Moreover, several more brain regions were found to be larger in one sex, with the majority being male dominant. Second to the SDN, the most intensely studied volumetric sex difference is that of the anteroventral periventricular (AVPV) nucleus which like the SDN is also just a collection of cells that are distinguished by Nissl, but unlike the SDN in this case there are more in the female than the male (Simerly et al 1985). Also unlike the SDN, there is a clear functional role for the AVPV in reproductive physiology. Neurons in this region project directly to the GnRH neurons that control the release of LH from the pituitary, which is under distinct control in males and females, showing a continuous pulsatile pattern in males versus a cyclic pattern marked by a large surge in females (Simerly 2002). A combination of GABAergic and dopaminergic neurons make up this nucleus and evidence suggests the relative survival of each is mediated by distinct mechanisms (Krishnan et al 2009, Waters \& Simerly 2009, Zup et al 2003). Moreover, the principal nucleus of the bed nucleus of the stria terminalis is also different in volume in males and females as a result of cell death in females, and there may be yet another distinct mechanism here involving epigenetic programming (Murray et al 2009). But it is hard to say with complete confidence that there are indeed multiple unique mechanisms as each incidence has been discovered and studied by a different group and thus no systematic comparisons have been made. 
The aforementioned hormonal modulation of dendritic spine synapses in hippocampal pyramidal neurons sparked interest in the potential for sex differences in dendritic morphology and synaptic patterning, and indeed several differences were found (Amateau \& McCarthy 2002a, Mong et al 2001, Schwarz et al 2008). When considering the sources of variability in synaptic patterning the obvious candidates to consider are the neurotransmitters that traffic at those same synapses. Indeed the notion that direct hormonal modulation of neurotransmitters and/or their cognate receptors must be the source of sex differences in brain and behavior preceded the discovery of many neuroanatomical sex differences. The number of studies exploring virtually every neurotransmitter or neuromodulator as a candidate target for hormonally mediated sexual differentiation are too numerous to review but can largely be summarized as demonstrating that too much or too little of any particular transmitter system was sufficient to disrupt normal sexual differentiation, but none of them were capable of substituting for the hormone. In other words, none of them were both sufficient AND necessary. While never clearly articulated, there was a general consensus that the process of sexual differentiation of the brain was so fundamental to reproductive fitness that it was likely to be highly redundantly organized so that the loss of no one system was capable of derailing the entire process, or directing it.

\section{Changing Views on the Origins of Brain Sex Differences}

The conclusion that sexual differentiation of the brain is multifactorial is essentially where the field stood until we made the surprising discovery in the early 2000's that prostaglandins, not neurotransmitters, were the primary target for hormonally mediated sexual differentiation of at least one system, the masculinization of the synaptic patterning of the medial POA and the expression of adult male sexual behavior (Amateau \& McCarthy 2004). We determined that estradiol, following aromatization from testosterone, up regulates expression of the cyclooxygenase genes, COX-1 and COX-2, leading to increased production of the prostaglandin PGE2. Normally associated with inflammation and fever, PGE2 is a membrane derived rapidly acting signaling molecule which binds to G-protein coupled receptors that are adenylate cyclase linked(Regan 2003). The subsequent production of cAMP within dendritic spines activates PKA (Wright \& McCarthy 2009) localized to dendritic spines leading to phosphorylation of the GluR2 subunit of glutamate AMPA receptors, thereby inducing trafficking of AMPA receptors to the membrane (Lenz et al 2011). In a manner not completely understood, this process induces the formation and stabilization of dendritic spine synapses (Lenz \& McCarthy 2010). The resulting density of spine synapses per unit of dendrite is twice as great in males, is stable throughout life and is highly correlated with the expression of adult male copulatory behavior measures such as frequency of and latency to mount a receptive female (Wright et al 2008). Most importantly, we found that injection of female pups with PGE2 into the brain during the sensitive period was capable of fully masculinizing the synaptic pattern and adult copulatory behavior in the absence of any neonatal hormone treatment (Amateau \& McCarthy 2004). This was the first report of a neurochemical other than steroids (testosterone or estradiol) that was both necessary and sufficient for masculinization of a neuroanatomical or behavioral endpoint. The effect of PGE2 was surprisingly specific, having no impact on the volume of the SDN, 
synaptic patterning in the hippocampus, amygdala or mediobasal hypothalamus and no effect on female sexual behavior, maternal behavior or emotionality (Todd et al 2005).

In addition to the effects of PGE2 being specific to neurons of the POA, we also detected an effect on the morphology of the local astrocytes. Males have more highly stellate astrocytes in the POA, with more numerous and more branched processes than those of females (Amateau \& McCarthy 2002b). Treatment of female neonates with PGE2 shifted the morphology of their astrocytes towards that of males and we have long speculated that the astrocytes are the source of the glutamate that activates the AMPA receptors that have been trafficked to the synaptic membrane (Wright et al 2010). Thus, we argued, there is a two-cell system inherent in sex differentiation that requires cross talk between neurons and astrocytes. But there was one observation that remained puzzling, a single injection of PGE2 to a newborn female rat pup was fully effective at masculinizing POA synaptic patterning and sexual behavior (Wright et al 2008). This surprising observation prompted us to consider the potential for a positively reinforcing system that was initiated by this single exposure to elevated PGE2 and the most likely candidate for that was a third cell type, the microglia.

Microglia are rather unfortunately named as they are not related to glia of the nervous system, the astrocytes and Schwann Cells. Unlike true glia which have common precursors with neurons, microglia are the primary immune cells of the brain and are modified macrophages that originate peripherally from the yolk sac and migrate into the brain in early embryonic development, where they take up permanent residence (Ginhoux et al 2013). Traditionally thought of as resting sentinels of the brain, microglia were believed to function primarily in the context of immune challenge or insult (Repovic \& Benveniste 2002, Streit 2000, Taylor \& Sansing 2013). Microglia both respond to and produce prostaglandins in response to an insult, along with other inflammatory and anti-inflammatory cytokines and growth factors (Streit 2000). It was this aspect of microglia function that presented itself as a potential mediator of a positively reinforcing effect of PGE2 and we found this to indeed be the case.

Microglia are highly variable in their morphology but this conveniently provides a window into their functional status. Ranging from a rounded, ameboid-like appearance to highly ramified and stellate (Figure 2), microglia morphology parallels a functional shift from "activated" to what was once called "quiescent" but is now more appropriately referred to as "surveying". The latter change is in light of the high degree of motility of ramified microglia which extend and retract their processes on a continual basis and in this way monitor a stable of nearby neurons for which they are responsible (Derecki et al 2013, Nimmerjahn et al 2005). Microglia are tiled relatively evenly throughout the brain leading to the belief that all neuronal populations are constantly being monitored, and perhaps assisted, by a local microglia. Moreover, if an injury does occur the local sentinels are the first to respond followed by recruitment of others and rapid proliferation (Neumann et al 2009, Taylor \& Sansing 2013). What is of interest in our system is that there is no injury, yet we found that the microglia in the male POA are more likely to exhibit an ameboid or rounded morphology and produce more PGE2 than those in the female (Lenz et al 2013). Moreover, when females are treated with either a masculinizing dose of estradiol, or PGE2, their 
microglia take on an activated morphology and increase the local production of PGE2, i.e. a positively reinforcing response. This additional PGE2 production is essential for both the establishment of the masculine synaptic pattern and adult sexual behavior. Thus it takes at least three cell types to properly masculinize the preoptic area; neurons, astrocytes and microglia, and the latter are of non-neuronal origin, even originating outside the brain (Lenz \& McCarthy 2014).

Microglia are being increasingly recognized as far more dynamic and engaged in brain development than previously thought (see(Nayak et al 2014). These unique cells provide both supportive and deleterious functions. Microglia regulate cell number by promoting cell survival through trophic factor release (Sierra et al 2010), influence synaptic plasticity and connectivity (Brown \& Neher 2014), and phagocytose apoptotic cells and debris (Neumann et al 2009). Interestingly, the ability of microglia to engulf other cells is not only limited to dying cells, but also to viable cells in a process termed "phagoptosis" (Brown \& Neher 2012, Brown \& Neher 2014). Targets of phagoptosis are stressed but otherwise viable cells, and are often recognized by the exposure of "eat me" signals on the surface of their membranes. The best characterized of these signals is phosphatidylserine (PS), a membrane phospholipid normally maintained in the inner leaflet, but reversibly redistributed to the external cellular surface under conditions of stress (Fadok et al 1992). Microglial phagocytosis is a critical regulator of postnatal neurogenesis (Sierra et al 2013, Sierra et al 2010), and impairments in phagocytic ability are thought to be involved in the progression of Rett Syndrome (Derecki et al 2013). We are detecting sex differences in the phagocytic profile of microglia in select brain regions (unpublished observation), however, the exact nature by which microglia sculpt behavior-specific circuitry remains to be elucidated.

\section{Sex differences in the brain are both a mosaic and canalized}

An unavoidable consequence of the multiplicity of mechanisms that mediate brain region specific sex differences is that there will be variability in the degree to which a region is either masculinized or feminized both across and within individuals. In our rodent animal models the majority of sex differences in the brain are induced by estradiol (McCarthy 2008), although androgens do directly impact some endpoints (Zuloaga et al 2008). It is conceivable that all end-points induced by estradiol and/or testosterone would involve the same signal transduction pathway and thus would be predicted to differentiate to the same degree within one individual and to the same degree across individuals if the hormone exposure is equivalent - a sort of "Unified Field Theory" of sex differences in the brain. But no matter how desirable such a scenario might be, it isn't true. Instead every brain region destined to differ in males and females appears to have co-opted a distinct mechanism for achieving that differentiation. This likely includes locally synthesized steroids, both androgens and estrogens, although this is vexingly difficult to definitively establish and quantify. Nonetheless, in one region estrogen receptors at the membrane interact with specific kinases critical to promoting sex-specific dendritic branching (Schwarz et al 2008), in another region the same receptors directly bind to DNA and up regulate transcription of prostaglandin producing genes (Amateau \& McCarthy 2004) or the GABA synthetic enzymes (Mong et al 2002) to ultimately control dendritic spine number after involving a number of other signaling molecules and cell types. 
The sheer number of nodal points of regulation guarantee diversity as there will be inherent genetic variation as well as environmentally induced epigenetic changes. Included in the genetic variation is the sex chromosome complement which will vary systematically in all male versus female cells but which may influence aspects of hormone action and the attendant signal transduction pathways in unique ways between brain areas (Arnold 2012, Arnold et al 2013). As a result each individual brain is a mosaic of regions of relative maleness or femaleness as well as regions that are unisex. Indeed one of the things that makes the brain so unique when considering sex differences is its capacity for a range of endpoints, from strongly male to strongly female and everything in between including sameness. The same cannot be said to be true for the reproductive tract, i.e. the gonads and genitalia, or the genes mediating sex differentiation, an observation codified as the $3 \mathrm{G}$ 's of sex differences by Daphna Joel (Joel 2012). There are no mosaics in these endpoints, the phenotypic or genotypic sex is purely binary, either male or female (with rare exceptions such as pseudo hermaphrodites, androgen insensitivity, etc).

In contrast, there is no true "male" or "female" brain, instead it is a mosaic. But a mosaic is not a blend. A blend would be a continuum of maleness to femaleness and if this were the case it would be evident in the data we have on sexually differentiated endpoints where we would see high variability within each sex and as a result there would be a low magnitude mean difference. Instead, just considering the data from this laboratory on sexually differentiated endpoints ranging from synaptogenesis (Amateau \& McCarthy 2002a, Amateau \& McCarthy 2004, Schwarz et al 2008), dendritic branching (Speert et al 2007), astrocyte (Amateau \& McCarthy 2002b, Mong et al 1996, Mong \& McCarthy 2002, Mong et al 2002) and microglia morphology (Lenz et al 2013) to neurogenesis (Bowers et al 2010, Waddell et al 2013), we consistently see a magnitude sex difference of one-to-two fold with low variability. Moreover, when we treat female pups with a masculinizing dose of either estradiol or testosterone, there is an increase or decrease in the endpoint that matches that of the male, but rarely if ever exceeds it. Likewise if we inject newborn male pups with either estradiol or androgen, which would provide even higher levels of steroid in the brain, there is usually no change or a response in the opposite direction. Put more simply, we do not observe a super-masculinization of either males or females when steroid levels are very high.

In summary, over many years of neuroanatomical quantification of various endpoints in the neonatal rodent brain we observe a consistent pattern in which there is a high degree of within sex consistency (i.e. low variability) and a high degree of consistency of the magnitude of the difference between the sexes. This could be because forces such as hormones or chromosome complement push the two sexes apart so that the mean response is distinctly different while other forces push the sexes together so that the response parameter stays within a range that does not differ too greatly between males and females.

Alternatively, there may be simple limitations to the degree of masculinization that are unrelated to sexual differentiation. For example, the number of motor neurons in the spinal nucleus of the bulbocavernosus is markedly higher in males than females as the function of these neurons is associated with penile erection (Forger et al 1992, Forger et al 1998, Freeman et al 1996). But you can't supermasculinize the number of motor neurons because 
they are rate limited by the number of muscle fibers, and therefore myogenesis is the limiting factor not sexual differentiation of the brain.

The above discussion is in many ways consistent with the evolutionary principle of "robustness", the ability of a species to buffer itself against minor environmental and genetic variation by maintaining a consistent phenotype. The term "canalization" was first coined by Waddington (Waddington 1959), and was used to explain how development almost always reaches the same endpoint despite external fluctuations or inherent noise. External fluctuations would include temperature, oxygen availability, $\mathrm{pH}$, nutrition, water salinity for aquatic species and so on, essentially anything that would cause stress to an organism by creating a disturbance in its predicted environment. Internal noise would be allelic or point mutation variation in genes, but could also include hormones. The concept of robustness is considered a universal emergent property of all living systems, but the degree to which canalization is a result of environmental versus genomic variation, and a combination thereof, remains a topic of debate (Posadas \& Carthew 2014). Waddington merged the two forces under the umbrella of epigenetics, arguing that external factors impacted genomics by silencing or promoting the expression of specific genes and thereby narrowing phenotypic variability. We here propose that the process of canalization contributes to both the divergence and convergence of sex differences in neuroanatomical endpoints. By reducing variability within a sex, sex differences are enhanced, but by restricting further divergence of phenotype, sex differences are reduced (Figure 3). Specific agents of canalization have been proposed and include organizing proteins, and non-coding RNAs such as microRNAs and transposons.

Empirical approaches to canalization are best achieved in rapidly reproducing species occupying an environment which can be controlled and manipulated and possessing a clearly quantifiable morphological endpoint that is subject to natural selection. Thus fish, drosophila and plants have been among the most commonly investigated. Heatshock protein 90 (Hsp90) is a central component of the "chaperome", a network of chaperone proteins that buffer the cell against physiological challenge and assist protein folding (Taldone et al 2014). Hsp90 is highly conserved and ubiquitously expressed in all cells. It has long been considered a potential master regulator of canalization (Ruden et al 2003) and this was definitively demonstrated to be the case for the control of eye size in a species of tilapia which rapidly progresses towards blindness when cave dwelling. This occurs because the water conditions found in caves inhibit Hsp90 resulting in expansion of eye size variability, generating both larger and smaller eyes than occurs in non-cave dwellers. Eyes are energetically expensive and since they are of no use to cave dwellers, smaller and smaller eyes are favored until they eventually disappear all together (Rohner et al 2013). If Hsp90 levels remain high there is very little variation in eye size and therefore nothing for selection to act upon even if the eyes are useless. Thus Hsp90 is a capacitor of morphological evolution. When Hsp90 is abundant phenotypic variation is suppressed, but when Hsp90 is low or absent, phenotypic variability increases immediately and provides a more varied substrate for selective pressure. This occurs in part because during a period of intense canalization genetic anomalies and mutations are allowed to accumulate and are then released from repression when canalization is relieved. Many of these mutations will be deleterious of course, but presumably some will not. 
The primary mechanism of Hsp90 and related chaperone proteins is to promote proper protein folding and function in the presence of minor genetic variation such as point mutations or premature truncations (Smith et al 2015). But an additional potential mechanism of canalization involves retrotransposons, originally called "jumping genes" when first discovered by Barbara McClintock but now referred to as mobile DNA elements. These ubiquitous and prevalent stretches of non-gene coding DNA are remnants of viral DNA insertions into the genome and while the overwhelming majority are silenced, a surprising number remain active and capable of transpositioning to new sites after production of RNA and key enzymes (see (Coufal et al 2009). LINE-1 is the most prevalent of the mobile DNA elements and is most active in neural progenitor and germ cell tissue (Belancio et al 2010, Singer et al 2010). Transposition events are highly variable between and within individuals and when they occur in neural progenitor cells are speculated to lead to genetic diversity between neurons within an individual. This process continues throughout life as a consequence of ongoing neurogenesis in the adult brain, and appears to be increased by external variables such as stress (Hunter et al 2014). Greater genomic variability in the brain, the reasoning goes, will provide greater flexibility in responding during challenging times (Erwin et al 2014). Others have taken the notion further and propose that transposition events contribute to phenotypic plasticity and hence provide physiological targets for natural selection, in essence an escape from canalization. Hsp90 suppresses transposon transposition by promoting expression of piwi-interacting RNAs, which are germ line-specific small regulatory RNAs known to maintain repetitive sequences and transposons in a repressed state (Piacentini et al 2014).

Another viable agent of canalization is a class of regulatory RNAs called microRNA's (miRNAs). As transcriptional regulators, miRNAs are perfectly poised to act as agents of canalization as their principle function is to dampen, not silence, gene expression (Posadas \& Carthew 2014). This is largely achieved by binding to messenger RNA's and either hastening their degradation or blocking translation. The balance between miRNAs and mRNAs determines the rate of transcription and this is particularly important during development. If miRNAs are inhibited there is increased variability of morphological endpoints, indicative of canalization. Moreover, within a certain range of transcription there is a lack of linearity between mRNA levels and protein output due to the governing effect of miRNAs, but if mRNA production outpaces miRNAs, i.e. all miRNAs are saturated, then linearity will be abruptly resumed (Figure 4) leading to switch-like or thresholding of regulatory networks (Posadas \& Carthew 2014). It is this thresholding aspect of miRNAs that makes them different from other regulators of gene expression such as co-expressors or co-repressors. The existence of a threshold is consistent with many sex differences in the brain that are hormone mediated. A central tenet of sexual differentiation is that males have a large surge in androgen production and local conversion of estradiol within the brain, but when steroid levels in the brain are measured one finds that females actually have considerable amounts of neural estrogens as well and in fact the difference from males is relatively small (Amateau et al 2004). Something must be altering the sensitivity of the female to her own steroids. This is most strikingly illustrated in our observation of neurogenesis in newborn rat pups. Males make twice as many new neurons as females during the first week of life and treating females with exogenous estradiol increases 
neurogenesis to that of males (Bowers et al 2010), yet endogenous estradiol measured in the hippocampus at that time is either not different in males and females (Konkle \& McCarthy 2011) or higher in females (Amateau et al 2004). So why are females making so many fewer new neurons than males? This mystery can be resolved by introduction of miRNAs as regulatory governors that suppress the proliferative effects of estradiol in females.

Consistent with this, a collection of miRNAs associated with proliferation were detected at higher levels in the developing hippocampus of females (Figure 5). Future studies will be essential to establishing a cause and effect relationship between miRNAs and canalization of hippocampal neurogenesis in males and females.

\section{Behavioral output may not match neuroanatomical canalization}

Rigidity of behavioral response in the face of variable stimuli is obviously a maladaptive strategy. Virtually all behavioral manifestation is the compilation of internal calculations of competing and converging inputs from multiple brain regions and each of those regions is in turn responding to multiple variables. Ultimately some neural pathway "wins" and a behavioral response is executed but outside of fixed action patterns, aspects of the behavior may vary in magnitude or frequency in subtle or obvious ways. One of the more vexing issues in the study of brain and behavior in the context of sex differences is causally connecting neuroanatomical variability with behavioral output. The SDN, arguably the largest neuroanatomical sex difference in the rodent brain, has been famously difficult to tie to a behavioral output. Analogous structures in humans, ferrets and sheep have been correlated with sexual partner preference (Baum 2006, LeVay 1991, Roselli et al 2004), but causality has not been established. Perhaps the best connection between neuroanatomy and behavior is bird song. There is in general a reasonable positive correlation between the degree that there is a sex difference in song behavior and the degree that there is a sex difference in the volume of key forebrain nuclei that control the learning and production of birdsong (Ball et al 2009), but even here the correlation does not always hold up. In StreakBacked Orioles the song control system shows the usual male bias in size yet females sing more than males (Hall et al 2010). In another instance involving songbirds, social status impacts the neuroanatomical sex difference but this does not map onto behavior (Voigt \& Gahr 2011). The relationship between neuroanatomy and behavior becomes even more difficult when considered at the synaptic level. The density of dendritic spines on POA neurons is correlated with the frequency of sexual mounting by males or experimentally masculinized females in rats (Wright et al 2008), but is it causal? Only by being able to selectively manipulate the density of dendritic spines selectively in this brain region without disturbing any other signaling pathways in this or other brain regions would one be able to address this question. But as explained above, the signal transduction pathway mediating the sex difference in synaptogenesis in the POA is a ubiquitous one, calling on such common mediators as protein kinase A (Wright \& McCarthy 2009). Thus even with the power of mouse genetics and conditional knockouts of select genes, it is still out of our grasp to so precisely manipulate one component of a specific circuit. This brings to mind a sentiment expressed by Per Södersten almost 30 years ago - "The search for morphological sex differences in adult rat brains that are caused by the 'organizing effect of perinatal androgen' and that can be related to sex differences in behavior has not been fruitful and 
may continue unrewarded" (Södersten 1987) - a sentiment that arguably still holds true today. But that is not because neuroanatomy has no impact on behavior but instead is likely due to the other tenet reiterated by Södersten and De Vries (De Vries \& Södersten 2009) that sex differences in behavior are context-dependent, which is just another way of saying that multiple inputs to multiple brain regions ultimately determine behavior. However we propose, as have many others, that the probabilities of specific behavioral outputs are shifted by the underlying neuroanatomy. If a portion of that anatomy is strongly canalized to be different in males and females, than the probability of a particular behavioral pattern would also be predicted to be shifted. Selection pressure is brought to bear on behavior, not on neuoranatomy directly, and therefore ipso facto if a neuroanatomical endpoint is canalized then it must be having a significant impact on behavior. Establishing if canalization is occurring may be easier to achieve then it first appears. Inhibition of the factors determining canalization during a critical developmental window, be they Hsp90, epigenetics or other regulatory elements would be predicted to abolish later sex differences in behavior by greatly increasing variability within each sex, i.e. reducing the probability that sex specific behavior will be expressed. And with this approach hopefully new advances in understanding brain and behavior relations can be made and avoid the further admonition of Södersten and De Vries that forging links between structure and function may be "akin to building a house of cards".

\section{Acknowledgments}

This work was supported by RO1MH52716-018, R01NS050525-08 and R01MH091424-02 to MMM.

\section{References}

Amateau SK, Alt JJ, Stamps CL, McCarthy MM. Brain estradiol content in newborn rats: sex differences, regional heterogeneity, and possible de novo synthesis by the female telencephalon. Endocrinology. 2004; 145:2906-17. [PubMed: 14988386]

Amateau SK, McCarthy MM. A novel mechanism of dendritic spine plasticity involving estradiol induction of prostglandin-E2. J Neurosci. 2002a; 22:8586-96. [PubMed: 12351732]

Amateau SK, McCarthy MM. Sexual differentiation of astrocyte morphology in the developing rat preoptic area. J Neuroendo. 2002b; 14:904-10.

Amateau SK, McCarthy MM. Induction of PGE(2) by estradiol mediates developmental masculinization of sex behavior. Nat Neurosci. 2004; 7:643-50. [PubMed: 15156148]

Arnold AP. The end of gonad-centric sex determination in mammals. Trends in genetics: TIG. 2012; 28:55-61. [PubMed: 22078126]

Arnold AP, Chen X, Link JC, Itoh Y, Reue K. Cell-autonomous sex determination outside of the gonad. Developmental dynamics: an official publication of the American Association of Anatomists. 2013; 242:371-9. [PubMed: 23361913]

Ball, GF.; Riters, LV.; MacDougall-Shackleton, SA.; Balthazart, J., editors. Sex differences in brain and behavior and the neuroendocrine control of the motivation to sing. Cambridge, UK: Cambridge University Press; 2009. p. 320-31.

Baum MJ. Mammalian animal models of psychosexual differentiation: When is 'translation' to the human situation possible? Horm Behav. 2006; 50:579-88. [PubMed: 16876166]

Baum MJ, Tobet SA, Cherry JA, Paredes RG. Estrogenic control of preoptic area development in a carnivore, the ferret. Cellular and molecular neurobiology. 1996; 16:117-28. [PubMed: 8743964]

Beach FA. Effects of gonadal hormones on urinary behavior in dogs. Physiology \& behavior. 1974; 12:1005-13. [PubMed: 4857634] 
Belancio VP, Roy-Engel AM, Pochampally RR, Deininger P. Somatic expression of LINE-1 elements in human tissues. Nucleic acids research. 2010; 38:3909-22. [PubMed: 20215437]

Bowers JM, Waddell J, McCarthy MM. A developmental sex difference in hippocampal neurogenesis is mediated by endogenous oestradiol. Biol Sex Differ. 2010; 1:8. [PubMed: 21208470]

Brager D, Sickel MJ, McCarthy MM. Developmental sex differences in calbindin-D28K and calretinin immunoreactivity in the neonatal rat hypothalamus. J Neurobiol. 2000; 42:315-22. [PubMed: 10645971]

Brown GC, Neher JJ. Eaten alive! Cell death by primary phagocytosis: 'phagoptosis'. Trends in biochemical sciences. 2012; 37:325-32. [PubMed: 22682109]

Brown GC, Neher JJ. Microglial phagocytosis of live neurons. Nature reviews. Neuroscience. 2014; 15:209-16. [PubMed: 24646669]

Coufal NG, Garcia-Perez JL, Peng GE, Yeo GW, Mu Y, et al. L1 retrotransposition in human neural progenitor cells. Nature. 2009; 460:1127-31. [PubMed: 19657334]

Davis EC, Popper P, Gorski RA. The role of apoptosis in sexual differentiation of the rat sexually dimorphic nucleus of the preoptic area. Brain Res. 1996; 734:10-18. [PubMed: 8896803]

De Vries GJ, Södersten P. Sex differences in the brain: the relation between structure and function. Hormones and behavior. 2009; 55:589-96. [PubMed: 19446075]

Derecki NC, Cronk JC, Kipnis J. The role of microglia in brain maintenance: implications for Rett syndrome. Trends in immunology. 2013; 34:144-50. [PubMed: 23122051]

Erwin JA, Marchetto MC, Gage FH. Mobile DNA elements in the generation of diversity and complexity in the brain. Nature reviews. Neuroscience. 2014; 15:497-506. [PubMed: 25005482]

Fadok VA, Voelker DR, Campbell PA, Cohen JJ, Bratton DL, Henson PM. Exposure of phosphatidylserine on the surface of apoptotic lymphocytes triggers specific recognition and removal by macrophages. Journal of immunology. 1992; 148:2207-16.

Forger NG, Hodges LL, Roberts SL, Breedlove SM. Regulation of motoneuron death in the spinal nucleus of the bulbocavernosus. Journal of neurobiology. 1992; 23:1192-203. [PubMed: 1469384]

Forger NG, Wagner CK, Contois M, Bengston L, MacLennan AJ. Ciliary neurotrophic factor receptor alpha in spinal motoneurons is regulated by gonadal hormones. The Journal of neuroscience: the official journal of the Society for Neuroscience. 1998; 18:8720-9. [PubMed: 9786979]

Freeman LM, Watson NV, Breedlove SM. Androgen spares androgen-insensitive motoneurons from apoptosis in the spinal nucleus of the bulbocavernosus in rats. Hormones and behavior. 1996; 30:424-33. [PubMed: 9047268]

Gilmore RF, Varnum MM, Forger NG. Effects of blocking developmental cell death on sexually dimorphic calbindin cell groups in the preoptic area and bed nucleus of the stria terminalis. Biol Sex Differ. 2012; 3:5. [PubMed: 22336348]

Ginhoux F, Lim S, Hoeffel G, Low D, Huber T. Origin and differentiation of microglia. Frontiers in cellular neuroscience. 2013; 7:45. [PubMed: 23616747]

Gorski RA, Gordon JH, Shryne JE, Southam AM. Evidence for a morphological sex difference within the medial preoptic area of the rat brain. Brain Res. 1978; 148:333-46. [PubMed: 656937]

Gorski RA, Harlan RE, Jacobson CD, Shryne JE, Southam AM. Evidence for the existence of a sexually dimorphic nucleus in the preoptic area of the rat. J Comp Neurol. 1980; 193:529-39. [PubMed: 7440781]

Hall ZJ, MacDougall-Shackleton SA, Osorio-Beristain M, Murphy TG. Male bias in the song control system despite female bias in song rate in streak-backed orioles (Icterus pustulatus). Brain, behavior and evolution. 2010; 76:168-75.

Hofman MA, Swaab DF. The sexually dimorphic nucleus of the preoptic area in the human brain: a comparative morphometric study. J Anat. 1989; 164:55-72. [PubMed: 2606795]

Hunter RG, Gagnidze K, McEwen BS, Pfaff DW. Stress and the dynamic genome: Steroids, epigenetics, and the transposome. Proceedings of the National Academy of Sciences of the United States of America. 2014

Jacobson CD, Gorski RA. Neurogenesis of the sexually dimorphic nucleus of the preoptic area in the rat. The Journal of comparative neurology. 1981; 196:519-29. [PubMed: 7217370] 
Joel D. Genetic-gonadal-genitals sex (3G-sex) and the misconception of brain and gender, or, why 3Gmales and 3G-females have intersex brain and intersex gender. Biol Sex Differ. 2012; 3:27. [PubMed: 23244600]

Kato Y, Nakashima S, Maekawa F, Tsukahara S. Involvement of postnatal apoptosis on sex difference in number of cells generated during late fetal period in the sexually dimorphic nucleus of the preoptic area in rats. Neuroscience letters. 2012; 516:290-5. [PubMed: 22521312]

Konkle AT, McCarthy MM. Developmental time course of estradiol, testosterone, and dihydrotestosterone levels in discrete regions of male and female rat brain. Endocrinology. 2011; 152:223-35. [PubMed: 21068160]

Krishnan S, Intlekofer KA, Aggison LK, Petersen SL. Central role of TRAF-interacting protein in a new model of brain sexual differentiation. Proceedings of the National Academy of Sciences of the United States of America. 2009; 106:16692-7. [PubMed: 19805359]

Lenz KM, McCarthy MM. Organized for sex - steroid hormones and the developing hypothalamus. The European journal of neuroscience. 2010; 32:2096-104. [PubMed: 21143664]

Lenz KM, McCarthy MM. A Starring Role for Microglia in Brain Sex Differences. The Neuroscientist: a review journal bringing neurobiology, neurology and psychiatry. 2014

Lenz KM, Nugent BM, Haliyur R, McCarthy MM. Microglia are essential to masculinization of brain and behavior. The Journal of neuroscience: the official journal of the Society for Neuroscience. 2013; 33:2761-72. [PubMed: 23407936]

Lenz KM, Wright CL, Martin RC, McCarthy MM. Prostaglandin E regulates AMPA receptor phosphorylation and promotes membrane insertion in preoptic area neurons and glia during sexual differentiation. PloS one. 2011; 6:e18500. [PubMed: 21490976]

LeVay S. A difference in hypothalamic structure between heterosexual and homosexual men. Science. 1991; 9:497-506.

McCarthy MM. Estradiol and the developing brain. Physiological reviews. 2008; 88:91-124. [PubMed: 18195084]

Mong JA, Kurzweil RL, Davis AM, Rocca MS, McCarthy MM. Evidence for sexual differentiation of glia in rat brain. Hormones and behavior. 1996; 30:553-62. [PubMed: 9047279]

Mong JA, McCarthy MM. Ontogeny of sexually dimorphic astrocytes in the neonatal rat arcuate. Dev Brain Res. 2002; 139:151-58. [PubMed: 12480129]

Mong JA, Nunez JL, McCarthy MM. GABA mediates steroid-induced astrocyte differentiation in the neonatal rat hypothalamus. J Neuroendo. 2002; 14:1-16.

Mong JA, Roberts RC, Kelly JJ, McCarthy MM. Gonadal steroids reduce the density of axospinous synapses in the developing rat arcuate nucleus: An electron microscopy analysis. J Comp Neurol. 2001; 432:259-67. [PubMed: 11241390]

Murray EK, Hien A, de Vries GJ, Forger NG. Epigenetic control of sexual differentiation of the bed nucleus of the stria terminalis. Endocrinology. 2009; 150:4241-7. [PubMed: 19497973]

Nayak D, Roth TL, McGavern DB. Microglia development and function. Annual review of immunology. 2014; 32:367-402.

Neumann H, Kotter MR, Franklin RJ. Debris clearance by microglia: an essential link between degeneration and regeneration. Brain: a journal of neurology. 2009; 132:288-95. [PubMed: 18567623]

Nimmerjahn A, Kirchhoff F, Helmchen F. Resting microglial cells are highly dynamic surveillants of brain parenchyma in vivo. Science. 2005; 308:1314-8. [PubMed: 15831717]

Orikasa C, Kondo Y, Usui S, Sakuma Y. Similar numbers of neurons are generated in the male and female rat preoptic area in utero. Neuroscience research. 2010; 68:9-14. [PubMed: 20538023]

Park JJ, Baum MJ, Paredes RG, Tobet SA. Neurogenesis and cell migration into the sexually dimorphic preoptic area/anterior hypothalamus of the fetal ferret. Journal of neurobiology. 1996; 30:315-28. [PubMed: 8807525]

Phoenix CH, Goy RW, Gerall AA, Young WC. Organizing action of prenatally administered testosterone proprionate on the tissues mediating mating behavior in the female guinea pig. Endocrinology. 1959; 65:369-82. [PubMed: 14432658] 
Piacentini L, Fanti L, Specchia V, Bozzetti MP, Berloco M, et al. Transposons, environmental changes, and heritable induced phenotypic variability. Chromosoma. 2014; 123:345-54. [PubMed: 24752783]

Posadas DM, Carthew RW. MicroRNAs and their roles in developmental canalization. Current opinion in genetics \& development. 2014; 27:1-6. [PubMed: 24791686]

Regan JW. EP2 and EP4 prostanoid receptor signaling. Life Sci. 2003; 74:143-53. [PubMed: 14607241]

Repovic P, Benveniste EN. Prostaglandin $\mathrm{E}_{2}$ is a novel inducer of oncostatin-M expression in macrophages and microglia. The Journal of neuroscience: the official journal of the Society for Neuroscience. 2002; 22:5334-43. [PubMed: 12097485]

Rhees RW, Shryne JE, Gorski RA. Onset of the hormone-sensitive perinatal period for sexual differentiation of the sexually dimorphic nucleus of the preoptic area in female rats. Journal of neurobiology. 1990a; 21:781-86. [PubMed: 2394991]

Rhees RW, Shryne JE, Gorski RA. Termination of the hormone-sensitive period for differentiation of the sexually dimorphic nucleus of the preoptic area in male and female rats. Dev Brain Res. 1990b; 52:17-23. [PubMed: 2331785]

Rohner N, Jarosz DF, Kowalko JE, Yoshizawa M, Jeffery WR, et al. Cryptic variation in morphological evolution: HSP90 as a capacitor for loss of eyes in cavefish. Science. 2013; 342:1372-5. [PubMed: 24337296]

Roselli CE, Larkin K, Resko JA, Stellflug JN, Stormshak F. The volume of a sexually dimorphic nucleus in the ovine medial preoptic area/anterior hypothalamus varies with sexual partner preference. Endocrinology. 2004; 145:478-83. [PubMed: 14525915]

Ruden DM, Garfinkel MD, Sollars VE, Lu X. Waddington's widget: Hsp90 and the inheritance of acquired characters. Seminars in cell \& developmental biology. 2003; 14:301-10. [PubMed: 14986860]

Schwarz JM, Liang S-L, Thompson SM, McCarthy MM. Estradiol induces hypothalamic dendritic spines by enhancing glutamate release: A mechanism for organizational sex differences. Neuron. 2008; 58:584-98. [PubMed: 18498739]

Sickel MJ, McCarthy MM. Calbindin D28-K immunoreactivity is a marker for a subdivision of the sexual dimorphic nucleus of the preoptic area in the rat: Developmental profile and gonadal steroid modulation. J Neurobio. 2000; 12:397-402.

Sierra A, Abiega O, Shahraz A, Neumann H. Janus-faced microglia: beneficial and detrimental consequences of microglial phagocytosis. Frontiers in cellular neuroscience. 2013; 7:6. [PubMed: 23386811]

Sierra A, Encinas JM, Deudero JJ, Chancey JH, Enikolopov G, et al. Microglia shape adult hippocampal neurogenesis through apoptosis-coupled phagocytosis. Cell stem cell. 2010; 7:48395. [PubMed: 20887954]

Simerly RB. Wired for reproduction: Organization and development of sexually dimorphic circuits in the mammalian forebrain. Annu Rev Neurosci. 2002; 25:507-36. [PubMed: 12052919]

Simerly RB, Swanson LW, Gorski RA. The distribution of monoaminergic cells and fibers in a periventricular preoptic nucleus involved in the control of gonadotropin release: immunohistochemical evidence for a dopaminergic sexual dimorphism. Brain Res. 1985; 330:5562. [PubMed: 2859086]

Singer T, McConnell MJ, Marchetto MC, Coufal NG, Gage FH. LINE-1 retrotransposons: mediators of somatic variation in neuronal genomes? Trends in neurosciences. 2010; 33:345-54. [PubMed: 20471112]

Smith HL, Li W, Cheetham ME. Molecular chaperones and neuronal proteostasis. Seminars in cell \& developmental biology. 2015

Södersten P. How different are male and female brains. TINS. 1987; 10:197-98.

Speert DB, Konkle ATM, Zup SL, Schwarz JA, Shiroor C, Taylor M. Focal adhesion kinase and paxillin: Novel regulators of brain sexual differentiation? Endocrinology. 2007; 148:3391-401. [PubMed: 17412802]

Streit WJ. Microglial response to brain injury: a brief synopsis. Toxicologic pathology. 2000; 28:2830. [PubMed: 10668987] 
Swaab DF, Hofman MA. Sexual differentiation of the human hypothalamus in relation to gender and sexual orientation. Trends in neurosciences. 1995; 18:264-70. [PubMed: 7571001]

Taldone T, Ochiana SO, Patel PD, Chiosis G. Selective targeting of the stress chaperome as a therapeutic strategy. Trends in pharmacological sciences. 2014; 35:592-603. [PubMed: 25262919]

Taylor RA, Sansing LH. Microglial responses after ischemic stroke and intracerebral hemorrhage. Clinical \& developmental immunology. 2013; 2013:746068. [PubMed: 24223607]

Todd BJ, Schwarz JM, McCarthy MM. Prostaglandin-E2: a point of divergence in estradiol-mediated sexual differentiation. Hormones and behavior. 2005; 48:512-21. [PubMed: 16126205]

Tsukahara S, Hojo R, Kuroda Y, Fujimaki H. Estrogen modulates Bcl-2 family protein expression in the sexually dimorphic nucleus of the preoptic area of postnatal rats. Neuroscience letters. 2008; 432:58-63. [PubMed: 18164816]

Tsukahara S, Kakeyama M, Toyofuku Y. Sex differences in the level of Bcl-2 family proteins and caspase-3 activation in the sexually dimorphic nuclei of the preoptic area in postnatal rats. Journal of neurobiology. 2006; 66:1411-9. [PubMed: 17013925]

Voigt C, Gahr M. Social status affects the degree of sex difference in the songbird brain. PloS one. 2011; 6:e20723. [PubMed: 21687671]

Waddell J, Bowers JM, Edwards NS, Jordan CL, McCarthy MM. Dysregulation of neonatal hippocampal cell genesis in the androgen insensitive Tfm rat. Hormones and behavior. 2013; 64:144-52. [PubMed: 23747829]

Waddington $\mathrm{CH}$. Canalization of development and genetic assimilation of acquired characters. Nature. 1959; 183:1654-5. [PubMed: 13666847]

Waters EM, Simerly RB. Estrogen induces caspase-dependent cell death during hypothalamic development. The Journal of neuroscience: the official journal of the Society for Neuroscience. 2009; 29:9714-8. [PubMed: 19657024]

Watson MA, Taylor H, Lephart ED. Androgen-dependent modulation of calbindin-D28K in hypothalamic tissue during prenatal development. Neuroscience research. 1998; 32:97-101. [PubMed: 9831256]

Woolley CS, McEwen BS. Estradiol mediates fluctuation in hippocampal synapse density during the estrous cycle in the adult rat. The Journal of neuroscience: the official journal of the Society for Neuroscience. 1992; 12:2549-54. [PubMed: 1613547]

Wright CL, Burks SR, McCarthy MM. Identification of prostaglandin E2 receptors mediating perinatal masculinization of adult sex behavior and neuroanatomical correlates. Dev Neurobiol. 2008; 68

Wright CL, McCarthy MM. Prostaglandin E2-induced masculinization of brain and behavior requires protein kinase A, AMPA/kainate, and metabotropic glutamate receptor signaling. The Journal of neuroscience: the official journal of the Society for Neuroscience. 2009; 29:13274-82. [PubMed: 19846715]

Wright CL, Schwarz JS, Dean SL, McCarthy MM. Cellular mechanisms of estradiol-mediated sexual differentiation of the brain. Trends in endocrinology and metabolism: TEM. 2010; 21:553-61. [PubMed: 20813326]

Zuloaga DG, Puts DA, Jordan CL, Breedlove SM. The role of androgen receptors in the masculinization of brain and behavior: what we've learned from the testicular feminization mutation. Hormones and behavior. 2008; 53:613-26. [PubMed: 18374335]

Zup SL, Carrier H, Waters EM, Tabor A, Bengston L, et al. Overexpression of bcl-2 reduces sex differences in neuron number in the brain and spinal cord. The Journal of neuroscience: the official journal of the Society for Neuroscience. 2003; 23:2357-62. [PubMed: 12657695] 


\section{Highlights}

- Sex differences in the brain are multifactorial in origin

- Non---neuronal cells are key players in masculinization of the brain

- The brain is a mosaic of maleness and femaleness

- The idea that sex differences in the brain are canalized is proposed 


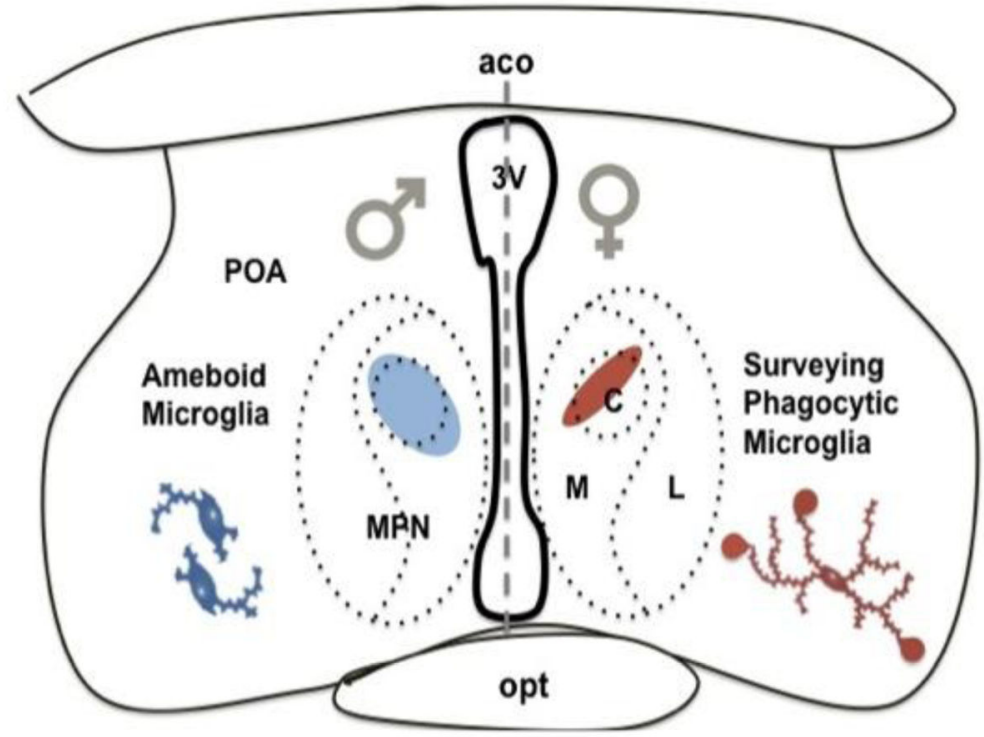

Figure 1. The Sexually Dimorphic Nucleus of the Preoptic Area

A collection of Nissl dense cells located in and around the Medial Preoptic Nucleus centralis (MPNc) constitutes the SDN. The volume of this nucleus is 3-5 times larger in males than females. Microglia morphology is also different in males and females in this brain region, being more rounded and ameboid-like in males and more ramified or "surveying" in females. $\mathrm{POA}=$ preoptic area, $\mathrm{MPN}=$ medial preoptic nucleus, $\mathrm{C}-$ central, $\mathrm{M}=$ medial, $\mathrm{L}=$ lateral, opt $=$ optic chiasm, aco $=$ anterior commissure, $3 \mathrm{~V}=$ third ventricle. 


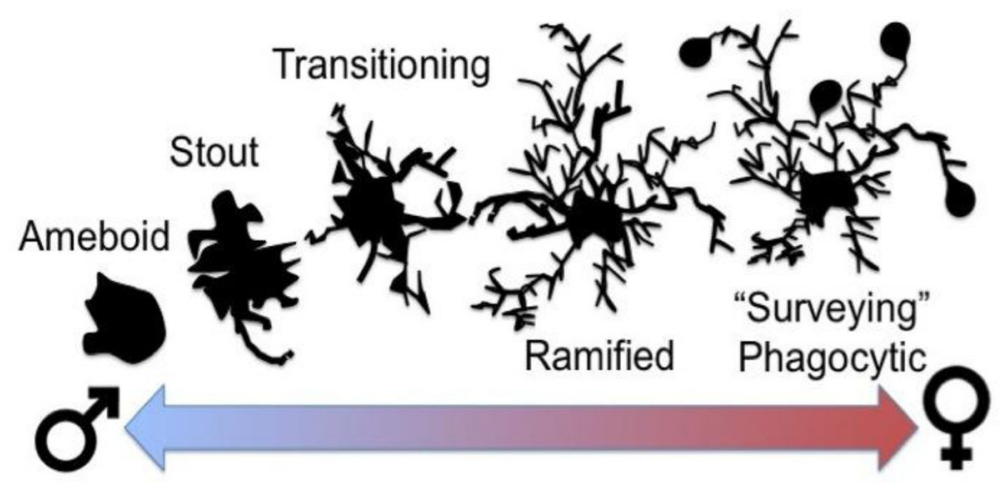

Figure 2. Sex differences in microglial morphology

Microglia vary in morphology from a rounded amoeboid like shape that is often indicative of activation to a highly ramified state previously considered quiescent but now known to be in a state of surveillance. In the developing preoptic area males have significantly more amoeboid shaped microglia while females have more surveying microglia (Lenz et al., 2013). 


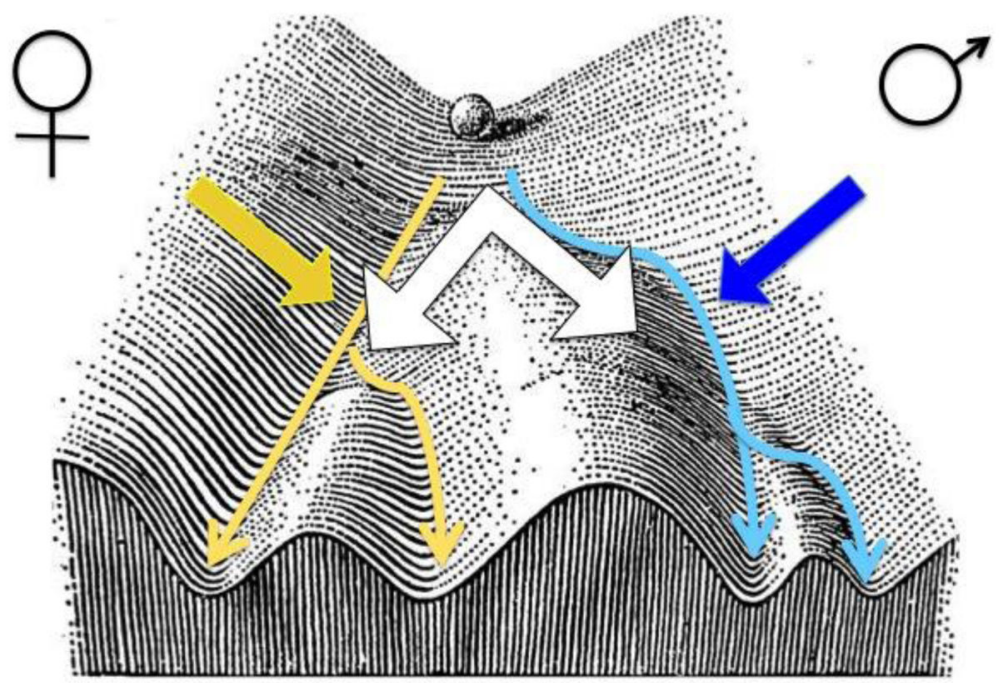

Figure 3. Canalization of sex differences in neuroanatomical endpoints

Canalization is a process first proposed by Waddington to explain the robustness of individuals in response to varying environments and genetic composition. Using Waddington's iconic image of the epigenetic landscape for illustration, we propose a similar process regulates neuroanatomical sex differences by reducing variability within each sex, thereby enhancing differences between the sexes, but also simultaneously assuring that the two sexes do not diverge too far apart from each other. Agents of canalization could include components of the chaperome, hormones, epigenetics and microRNAs (see text for details). 


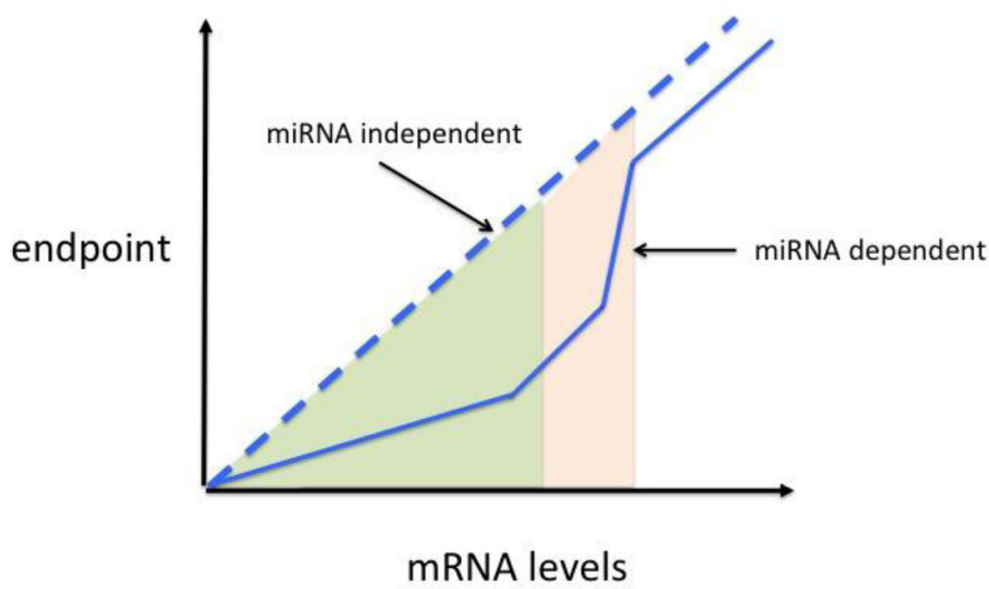

Figure 4. MicroRNA's may mediate canalization

MicroRNAs (miRNA) are governors of gene expression by inhibiting or degrading mRNA's. In the absence of miRNA regulation, the relationship between mRNA and protein synthesis is predicted to be linear. However under conditions of miRNA regulation low levels of mRNA are effectively governed and expression is repressed at a wide range of mRNA concentrations. If the mRNA level saturates the capacity of the miRNA, there is a rapid rise in expression and eventual return to linearity, thus creating a threshold below which there is little response and above which there is maximal response. The same principle could be applied to neuroanatomical endpoints such as dendritic spine density or cell genesis, processes which require protein synthesis (redrawn from (Posadas \& Carthew 2014). 


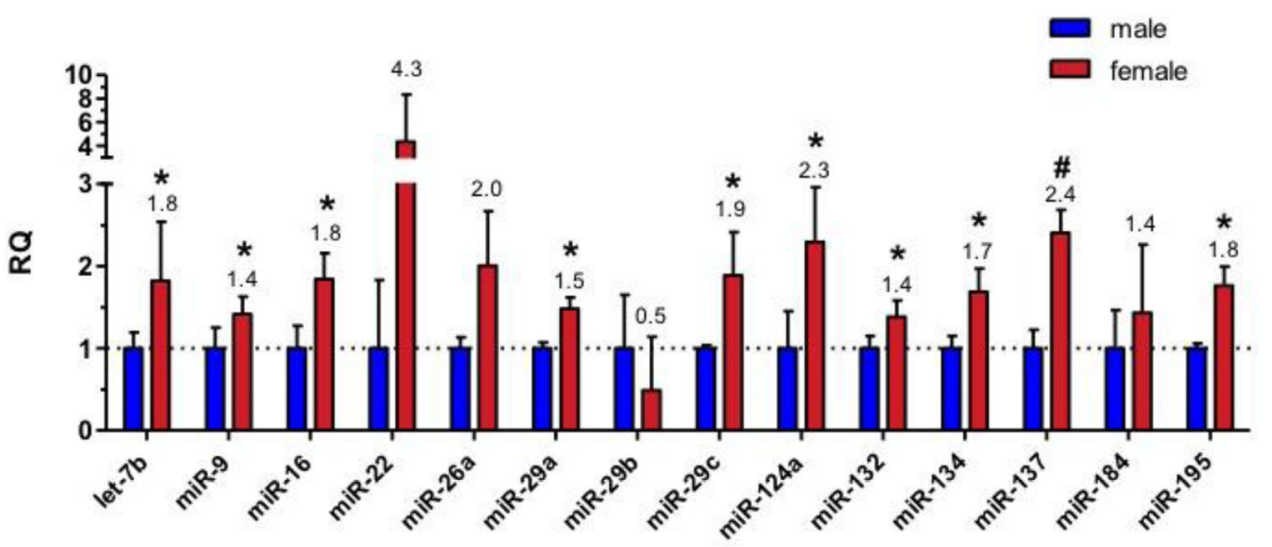

Figure 5. Sex differences in microRNA's in the developing hippocampus

Sex differences in expression levels of selected microRNAs in the dentate gyrus of Sprague Dawley rat pups during the first postnatal week, as determined via quantitative PCR (qPCR). Total RNA was isolated from dentate gyrus of untreated male and female pups on postnatal day 4 and transcribed to cDNA, and qPCR of 14 selected mature miRNAs was performed using custom TaqMan MicroRNA Array Cards (Life Technologies). Relative quantification (RQ) levels are normalized to U6 and sno6 small RNAs and expressed relative to males for each target miRNA. $\mathrm{n}=4$ for each sex (ANOVA; * $\mathrm{p}<0.05$; $\# \mathrm{p}<0.001)$. 